\title{
Energy Efficient Hierarchical Clustering Mechanism for Wireless Sensor Network Fields
}

\author{
Hamid Ali Abed Alasadi \\ Professor, \\ Computer Science Departmentt, \\ Faculty of Education for Pure Science \\ Basra University, Iraq
}

\begin{abstract}
In wireless sensor networks it becomes infeasible to recharge or substitute the dead batteries of the sensor nodes. As soon as, some of the sensor nodes in a Wireless Sensor Network (WSN) run out of energy, they stop functioning initiating progressive deconstruction of the network. Hence, each and every protocol should be so designed in such a way that minimum energy should be expended during sensing, processing and communication. This work suggests the development of an enhanced hierarchical clustering method, the Energy Efficient Hierarchical Clustering Mechanism (EEHCM) for wireless sensor network fields. This is a welldistributed clustering mechanism and the cluster head selection is based on the residual energy, communication cost and the distance to the base station. The main distinguishing feature of the proposed algorithm is that the cluster head selection is accomplished in mere few steps and its hierarchical nature. Simulation results clearly display that the proposed EEHCM scheme depicts an excellent reduction in communication energy and backbone energy consumption. Also, the energy efficiency in EEHCM is enhanced to a great extent. It is noted that the first node death and the last node death are delayed, and hence the overall network lifetime is prolonged.
\end{abstract}

\section{General Terms}

Wireless sensor network, cluster head and energy efficiency.

\section{Keywords}

Base station, routing efficiency, clustering efficiency, network lifetime, distributed clustering.

\section{INTRODUCTION}

A wireless sensor node contains low power processor, a tiny memory, a radio frequency module, sensing devices and limited powered batteries. Much of the energy consumption takes place through wireless communication. An effectual way to lessen energy usage is to group the sensor nodes into numerous clusters and each individual cluster has a leader referred as cluster head [1]. The cluster head forwards the aggregated data to base station. In distributed clustering, the cluster head changes from one node to another node based on few parameters. Since, these wireless sensor nodes are power constrained devices, long distance transmissions should be generally kept minimum in order to enlarge the network lifetime. Thus, the direct communications between wireless sensor nodes and the base station (BS) are not extremely fortified. An effectual procedure to perk up efficiency, is by ordering the sensor network into numerous clusters, with each of the cluster electing one node as its leader or the cluster head.

The accumulated data will then be transmitted to the base station directly or by multi-hop fashion by the cluster head
$(\mathrm{CH})$. In such an organization, only cluster heads are mandatory to transmit the data over longer distances. The remaining nodes will want to carry-out only short-distance transmission. Clustering methodology is basically classified into centralized, distributed and hybrid clustering methodologies. Hierarchical methodology could be employed for all these clustering mechanisms [2].

When energy efficiency is a key criterion during clustering, hierarchical methodology could be additionally effective. The cluster heads all over the wireless sensor network will be distributed into different levels or hierarchy or tier. First level cluster heads will hand-over the aggregated data to the second level cluster heads. The second level cluster heads will handover the data to third level cluster heads. The cluster head at the final level only will be forwarding every data to the base station. By following this hierarchical method, energy wastage can be avoided to a greater extent. This research work gives a deep description about Energy Efficient Hierarchical Clustering Mechanism (EEHCM) for effectual formation of clusters in wireless sensor network.

The paper has been prearranged as follows. An overview of wireless sensor network, their features and clustering in WSN has been entailed in Section 1. The existing hierarchical clustering methodologies have been discussed in section 2 . The restrictions of the existing methodologies and the features of the proposed methodology has been discussed in Section 3 and finally the last Section 4 gives the conclusion.

\section{EXISTING CLUSTERING METHODS}

The major hierarchical clustering algorithms for wireless sensor network are Low Energy Adaptive Clustering Hierarchy (LEACH), Threshold sensitive Energy Efficient Network (TEEN) and Scaling Hierarchical Power Efficient Routing (SHPER). LEACH is a clustering mechanism that generally distributes the energy consumption all along its network, the network being separated into minor clusters and $\mathrm{CHs}$ which are virtuously distributed in manner and the indiscriminately elected $\mathrm{CHs}$, collect the information from the sensor nodes which are coming under its cluster. The LEACH protocol includes four chief steps for each round: the advertisement phase, the cluster set-up phase, the schedule creation phase and the data transmission phase. During the advertisement phase, the appropriate $\mathrm{CH}$ nodes will be conveying a notification to the nodes coming under them to convert as a cluster member in its respective cluster. The sensor nodes will be accepting the offer based on the received signal strength (RSS). In cluster set-up phase, the sensor nodes will be responding to their selected cluster heads. In schedule creation phase, as the cluster head receives response from nodes it have to construct a TDMA time-slot and send it back to the cluster members to intimate them when they have to pass the data to it. In data transmission stage, the data 
collected by the discrete sensors will be given to the $\mathrm{CH}$ during their corresponding time intervals. The chief restriction here is that, the radio of the cluster members will be turnedoff to lessen the energy consumption after the data transmission during particular time-slot is finished. Here, in LEACH clustering protocol the multi-cluster interference problem was solved by using a unique CDMA codes for each cluster. The energy drain is banned for the same wireless sensor nodes which have been selected as the cluster leader by randomization, for each time the cluster head would be altered. The $\mathrm{CH}$ is accountable for assembling data from the cluster members and fusing it. Finally, each cluster head will be dispatching the fused data to the base station. LEACH shows a substantial improvement mainly in terms of energy efficiency [3].

The preliminary stage of TEEN protocol is the establishment of clusters. In this step, every cluster member nodes develops as a cluster head for a certain time interval mentioned as the cluster period. The SHPER protocol comprises of the base station and the wireless sensor nodes which are arbitrarily detached over the restricted region of attention. The base station and all the wireless sensor nodes are found to be normally stationary. The end users can access the information from the base station which is logically positioned far away from the sensing field. Every cluster nodes are assembled together into distinct and multiple clusters. Within every cluster, one node is allocated to be the cluster head. The cluster head election process in SHPER is virtuously based on the residual energy. The cluster heads that are nearer to the base station and which could correspond with the base station with the rational power utilization is considered to be the highest level cluster head. Likewise, the cluster head which is usually located far away from the base station is considered to be the lowest level cluster head.

The functioning of the SHPER protocol comprises of two main phases i.e. the initialization phase and the steady state phase. Throughout the initialization phase, the base station decides which node should be the cluster head. The nodes rather than the cluster head develops as the member nodes. Each cluster head along with some cluster nodes are assembled together to form precise clusters. The base station sends the ID of each $\mathrm{CHs}$ which are freshly selected. Furthermore, each sensor node chooses the cluster to which it fits and informs its cluster head on being the member of that particular cluster. The cluster head notifies the member nodes concerning the time when they have to transmit the information. Consequently, the data is collected by the cluster head and then aggregated, further being transmitted to the base station during the steady state phase [4].

LEACH protocol is less-effective when intervallic transmissions are needless, thus producing unusable power consumption. The selection of cluster head is purely based on priority, and hence there is a choice that the weaker nodes to be drained, when they are nominated as cluster heads as regularly as the stronger wireless sensor nodes. Furthermore, the protocol is based on the suppositions that, every sensor nodes start with identical energy capacity in every election round and all the wireless sensor nodes can transmit with satisfactory power to the base station if desirable. Nevertheless, in frequent cases these conventions are found to be unrealistic [5-9].

TEEN protocol has been recognized for reactive networks, so as to take action for unexpected variations in the sensed attributes. TEEN is apt for time critical applications and not proper for applications where periodic reports are required. In case of SHPER protocol, the election of cluster head is only based on the base station. Hence needless transmissions happen between the base station and the cluster heads. Also, the base station should keep track on the wireless sensor nodes so as to choose which node has the highest residual energy, thereby causing augmented power consumption $[10,11]$.

\section{THE PROPOSED EEHCM SYSTEM}

In case of the existing clustering techniques, the election of $\mathrm{CHs}$ and the cluster nodes are completely carried out by the base station. Thereby, they are disposed to additional power consumption. The proposed method mainly considers that the cluster head as to be totally accountable for all the process including the election of new cluster heads and the member nodes. The cluster head computes the power expended by the sensor nodes, which commonly depends on the obtainable power at the sensor nodes, and the distance between sensor nodes and the cluster head.

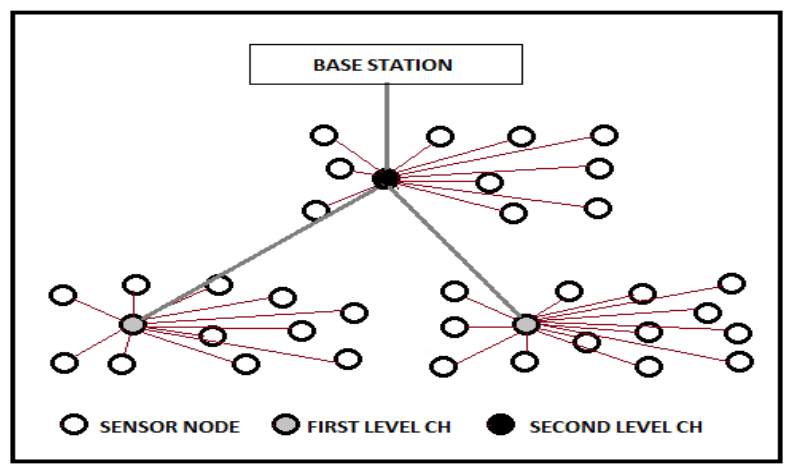

Figure 1: Hierarchical Clustering Architecture of EEHCM

Here, two different thresholds are employed namely the hard threshold and the soft threshold to further reduce the number of transmissions during data aggregation. Generally, hard threshold is the smallest conceivable values of an attribute to trigger a sensor node to switch-on its transmitter and then transmit the data to the cluster head. Soft threshold is a slight change in the value of the sensed attribute that triggers the sensor node to switch-on its transmitter and transmit the data. The former tries to lessen the number of transmission by letting the sensor nodes to transmit only when the sensed attribute is ahead of the particular critical value. Likewise, this soft threshold additionally trims-down the number of transmissions when there is minor change or no change in the value of the sensed attribute. For each cluster change, the values of both these threshold levels could be changed, thereby enabling the user to accomplish the adjustment between energy efficiency and data accuracy. The sensor nodes transmit the sensed data to the cluster head.

The chief distinctive feature of this method is that, the residual energy is transmitted along with the sensed data by the sensor nodes to the cluster head. The cluster head merely transmits the aggregated data to the base station. Figure 1 shows the hierarchical clustering architecture of the proposed EEHCM distributed clustering methodology. Every procedures like initialization, the formation of clusters, the election of cluster heads and the monitoring the residual energy is done solely by the cluster head. Store and forward procedure is followed at the cluster head, so that the sensed attribute along with the residual energy is composed from the cluster nodes, stored at the cluster head and further, only the aggregated information is forwarded to the base station. Since, 
the base station has no direct-link with the cluster nodes, needless transmissions are avoided, thereby lessening enormous energy consumption. As defined in the earlier sections, the cluster nodes need to be reliably distributed over the entire sensor network for sinking the energy utilization. In the anticipated EEHCM clustering methodology, the redundant formation of cluster heads is greatly evaded. The proposed EEHCM clustering methodology includes set-up phase and steady state phase. Figure 2 represents the timeline concept of the proposed distributed clustering methodology.

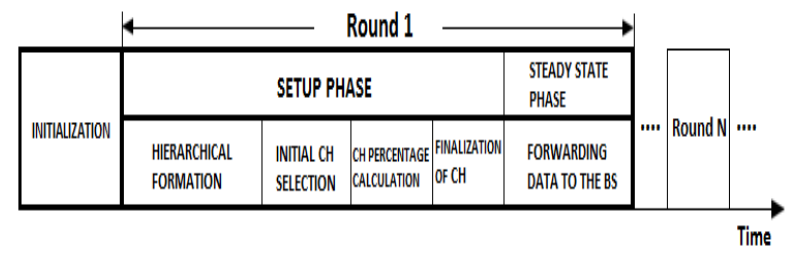

Figure 2: Timeline Concept of the Proposed Methodology

The chief activities during the set-up phase are the hierarchical layer formation, election of candidate nodes, initial selection of cluster heads, scheduling at each cluster, calculating the percentage of cluster heads, finding cluster head for $\mathrm{CH}$-to- $\mathrm{CH}$ data transmission and finalization of the $\mathrm{CH}$. During this set-up phase, every node originally decides whether it could develop as a candidate node for the current round. An advertisement message has been employed to elect the cluster heads. Each and every candidate nodes broadcast an advertisement message within its radio transmission limit. The advertisement range is twice the maximum distance to cover each and every other levels. This choice is based on the current strength of the signal of the advertisement message. When all the wireless sensor nodes have decided their individual clusters, the wireless sensor nodes start transmitting its data to their appropriate cluster heads. The cluster head on getting every messages from the wireless sensor nodes that likes to be assimilated in the cluster, and based on the number of wireless sensor nodes contained in the cluster, the cluster head generates a schedule and allocates every node with a time-slot for data transmission.

For this sole reason, every cluster head makes the use of twoway handshake technique containing messages like Request (REQ) and Acknowledgement (ACK). Each and every cluster head generally broadcasts a REQ message within its own advertisement range. When the cluster head accepts this REQ message, it then transmits an ACK message back to the cluster head that has transmitted the REQ message. The wireless sensor node that has transmitted REQ message on receiving the ACK message, it selects this cluster head which transmitted the ACK message as the next successive hop. If the cluster head could not treasure the upward cluster head it additionally chooses the base station as the next hop.

In EEHCM, steady state phase is analogous to all other cluster-based systems. The chief activities done in this phase are sensing and the transmission of the sensed information. Each sensor node does sensing operation and transmits the data to its respective cluster head during its assigned time schedule. When every data has been received, the cluster head carries-out data aggregation so as to moreover reduce the amount of data for communication. Each cluster head lastly transmits the aggregated data to the base station along the $\mathrm{CH}$ to-CH routing path, which has been constructed during the set-up phase. After every data has been transmitted, the network proceeds back to set-up phase again, and the next successive round begins by electing fresh candidate nodes.
Table 1: Simulation Parameter Setup

\begin{tabular}{|l|c|}
\hline \multicolumn{1}{|c|}{ Parameter } & Values \\
\hline Topology & $500 \times 500 \mathrm{~m}^{2}$ \\
\hline Number of nodes & 30 \\
\hline Data packet size & 4000 bytes \\
\hline Control packet size & 550 bytes \\
\hline Initial energy & 1 Joule/Node \\
\hline Transmitter power & $31.32 \mathrm{~mW}$ \\
\hline Receiver power & $35.28 \mathrm{~mW}$ \\
\hline Ideal power & $712 \mathrm{~mW}$ \\
\hline Sleep power & $144 \mathrm{~mW}$ \\
\hline
\end{tabular}

Table 2: Simulated values for Energy consumption

\begin{tabular}{|c|c|c|c|c|c|c|c|c|}
\hline \multicolumn{2}{|c|}{$\begin{array}{l}\text { Number of } \\
\text { Rounds }\end{array}$} & \multirow{2}{*}{ 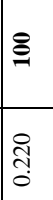 } & \multirow{2}{*}{\begin{tabular}{|l|} 
క్ \\
0 \\
0 \\
0
\end{tabular}} & \multirow{2}{*}{$\begin{array}{l}\stackrel{\bar{\sigma}}{\Xi} \\
\stackrel{\circ}{\circ} \\
\end{array}$} & \multirow{2}{*}{  } & \multirow{2}{*}{$\frac{\text { ثั }}{\frac{8}{0}}$} & \multirow{2}{*}{ 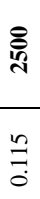 } & \multirow{2}{*}{  } \\
\hline 可 & $\begin{array}{c}\text { LEA } \\
\text { CH }\end{array}$ & & & & & & & \\
\hline  & $\begin{array}{c}\text { EEH } \\
\text { CM }\end{array}$ & $\frac{n}{0}$ & $\frac{2}{3}$ & ô & $\frac{8}{0}$ & ô & $\begin{array}{l}\text { oे } \\
\text { o. }\end{array}$ & $\begin{array}{l}0 \\
\vdots \\
0 \\
0\end{array}$ \\
\hline
\end{tabular}

The performance of the proposed EEHCM clustering methodology has been evaluated through simulations for 30 wireless sensor nodes and the results have been compared with LEACH. All the simulations have been executed using the network simulator (NS-2). Table 1 demonstrates the desirable simulation parameters. The sensor nodes are deliberated to be immobile (stationary) with the uniform initial energy level of $1 \mathrm{~J} /$ Node. The wireless sensor nodes are thoroughly prepared with every possible power control abilities. The base station has adequate energy and energy scarcity does not occur at any cost.

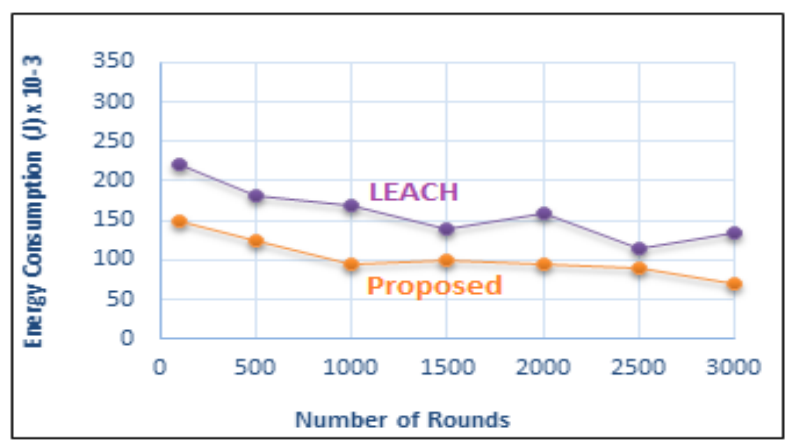

Figure 3: Total energy consumption in LEACH and EEHCM

Table 3: Simulated values for Lifetime comparison

\begin{tabular}{|c|c|c|c|c|c|c|c|c|}
\hline \multicolumn{2}{|c|}{ Number of Rounds } & \multirow{2}{*}{$\begin{array}{l}\text { ఏ } \\
90\end{array}$} & \multirow{2}{*}{$\begin{array}{l}\stackrel{\text { in }}{ } \\
53\end{array}$} & \multirow{2}{*}{$\begin{array}{l}\stackrel{\Xi}{\Xi} \\
40\end{array}$} & \multirow{2}{*}{$\begin{array}{l}\stackrel{8}{2} \\
20\end{array}$} & \multirow{2}{*}{$\frac{\stackrel{\text { తి }}{4}}{4}$} & \multirow{2}{*}{ 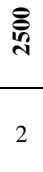 } & \multirow{2}{*}{$\begin{array}{l}\text { ఏ్ } \\
\\
\end{array}$} \\
\hline$\%$ & $\begin{array}{c}\text { LEA } \\
\text { CH }\end{array}$ & & & & & & & \\
\hline Diretime & $\begin{array}{c}\text { EEH } \\
\text { CM }\end{array}$ & 95 & 70 & 62 & 46 & 28 & 23 & 19 \\
\hline
\end{tabular}




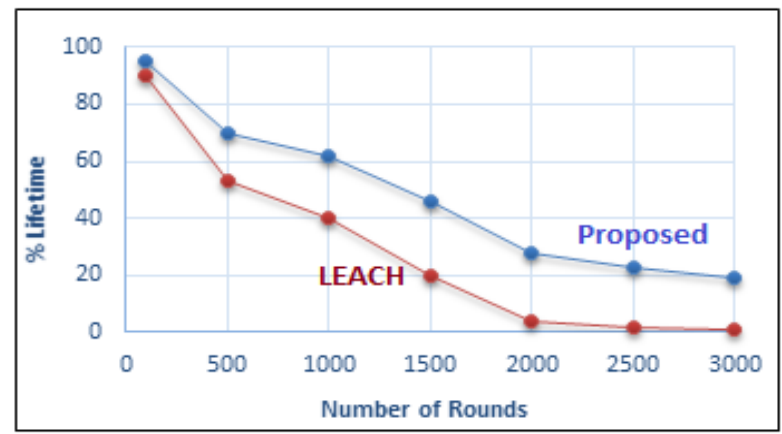

Figure 4: Percentage Lifetime in LEACH and EEHCM

Figure 3 shows the total energy consumption for every round for both LEACH and EEHCM. Primarily, at 100 rounds the total energy consumption is 0.22 Joules and 0.15 Joules for LEACH and EEHCM correspondingly. Similarly, for 3000 rounds, the energy consumption is 0.135 Joules and 0.07 Joules for LEACH and EEHCM correspondingly. The average energy consumption is 0.160 Joules for LEACH and 0.104 Joules for EEHCM. EEHCM displays 35\% reduced energy consumption in comparison to LEACH. This is, since the proposed EEHCM procedure decreases needless making of cluster heads and exploits the $\mathrm{CH}$-to- $\mathrm{CH}$ routing path, and thus unnecessary energy utilization is evaded.

Figure 4 illustrates the percentage lifetime for particular number of rounds for both LEACH and EEHCM. Primarily, in 100 rounds the percentage lifetime of LEACH is $90 \%$ and that of EEHCM is $95 \%$. Correspondingly, in 3000 rounds, the percentage lifetimes of LEACH and EEHCM are 1\% and 19\% respectively. At an average, EEHCM displays 19\% enhancement in lifetime when compared to LEACH. This undoubtedly displays that the proposed EEHCM method has enhanced lifetime when compared to LEACH, because of the hierarchical ideas engaged in the proposed methodology.

Table 4: Simulated values for Backbone energy comparison

\begin{tabular}{|c|c|c|c|c|c|c|c|c|}
\hline \multicolumn{2}{|c|}{ Number of Rounds } & ¿্ণ & \&ั & ¿ & ర్గ్రి & চి & ర్ & ర్లి \\
\hline \multirow{2}{*}{ 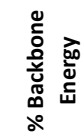 } & LEACH & 30 & 27 & 25 & 24 & 22 & 20 & 17 \\
\hline & EEHCM & 20 & 19 & 15 & 14 & 12 & 11 & 12 \\
\hline
\end{tabular}

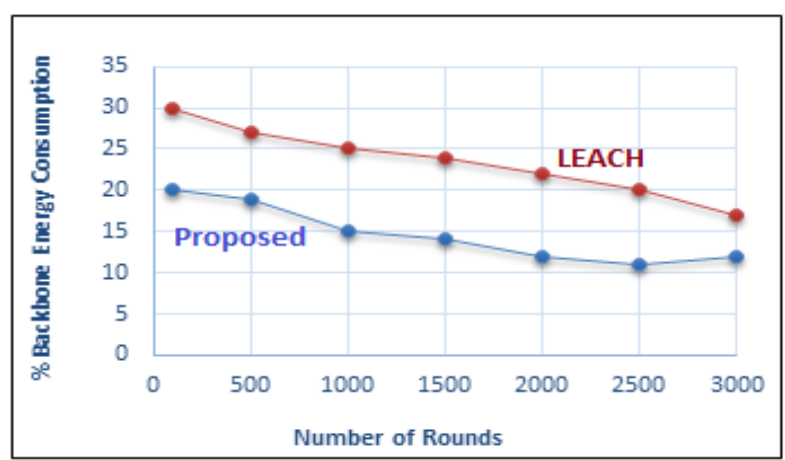

Figure 5: Backbone Energy Consumption in LEACH and EEHCM

Figure 5 illustrates the percentage backbone energy consumption against the number of rounds for both LEACH and EEHCM. The backbone energy consumption is smaller in EEHCM when compared to LEACH for all the successive rounds. The average percentage backbone energy consumption in LEACH and EEHCM is $23.57 \%$ and $14.71 \%$ correspondingly. EEHCM displays $37.59 \%$ reduced backbone energy consumption when compared to LEACH. This is mostly because LEACH uses arbitrary $\mathrm{CH}$ selection mechanism and the direct forwarding of data to the base station by the cluster head. But EEHCM employs hierarchical technique for cluster formation and the aggregated data is not unswervingly forwarded to the base station. Thus, it could be clearly seen that the proposed methodology is tremendously efficient in terms of backbone energy consumption when compared to LEACH.

Figure 6 entails the energy efficiency assessment of both EEHCM and LEACH. Primarily, in 100 rounds the energy efficiency is $42 \%$ and $69 \%$ for LEACH and EEHCM correspondingly. Likewise, in 3000 rounds the energy efficiency is $20 \%$ and $49 \%$ correspondingly for LEACH and EEHCM. All over the process, energy efficiency is improved in EEHCM when compared to LEACH.

Table 5: Simulated values for Energy efficiency comparison

\begin{tabular}{|c|c|c|c|c|c|c|c|c|}
\hline \multicolumn{2}{|c|}{ Number of Rounds } & 100 & 500 & 1000 & 1500 & 2000 & 2500 & 3000 \\
\hline \multirow{2}{*}{ 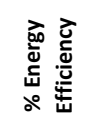 } & LEACH & 42 & 45 & 43 & 46 & 38 & 27 & 20 \\
\hline & EEHCM & 69 & 67 & 64 & 67 & 61 & 55 & 49 \\
\hline
\end{tabular}

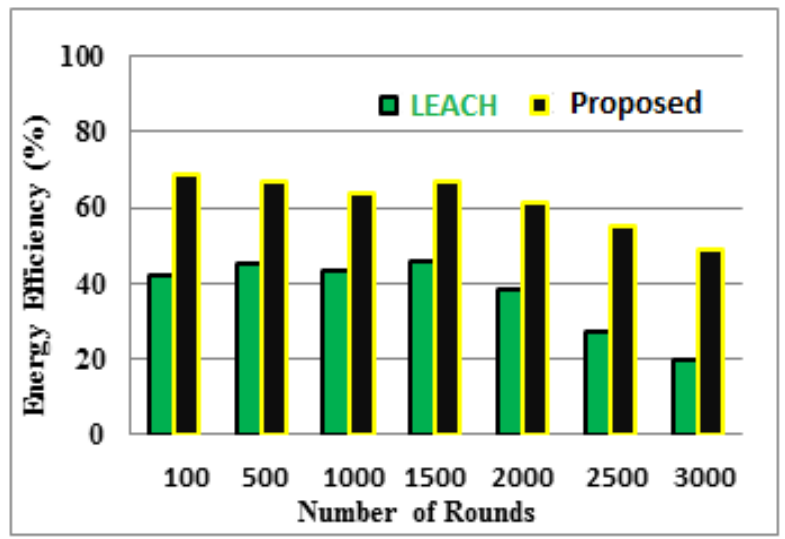

Figure 6: Energy Efficiency Comparison in LEACH and EEHCM

Thus, it could be noticeably understood that the proposed EEHCM mechanism is a well-distributed and energy-efficient clustering mechanism, and could be engaged for successfully clustering of the wireless sensor nodes in wireless sensor network.

\section{CONCLUSION}

Clustering is the most noteworthy issue for networks where resources are limited. Thus, it is mandatory to make use of the available energy efficiently. There becomes a need in wireless sensor network to successfully group the sensor nodes in to clusters. This paper is anxious with the proposal of EEHCM methodology for static wireless sensor network. This method employs hierarchical architecture for cluster formation in a wireless sensor network. The strange feature of this technique, 
compared to the existing techniques is that the election of the cluster head, the cluster nodes and the monitoring of residual energy is virtuously done by the cluster head. As the base station does not encompass in these processes, unnecessary energy wastage for long distances communication is evaded, thus dropping-down the energy usage to a considerable extent. Simulation results noticeably show that the proposed EEHCM methodology portrays an excellent reduction in backbone energy consumption and total energy consumption. However, the energy efficiency in EEHCM is improved to a great extent. It is prominent that the first node death and the final node death are significantly delayed thereby the overall lifetime of the wireless sensor network is amended by the proposed EEHCM methodology.

\section{REFERENCES}

[1] Heinzelman, W., Chandrakasan, A., Balakrishnan, H., 2000, "Energy- Efficient Communication Protocol for Wireless Microsensor Networks", Proceedings of the 33rd Hawaii International Conference on System Sciences.

[2] Manjeshwar, A., and Agarwal, 2001, "TEEN: A Routing Protocol for Enhanced Efficiency in Wireless Sensor Networks", Issues in Wireless Networks and Mobile Computing, San Francisco, USA.

[3] Youssef, A., Younis, M., Youssef, M., and Agrawala, A., 2006, "Distributed Formation of Overlapping Multi-Hop Clusters in Wireless Sensor Networks", Proceedings of the 49th Annual IEEE Global Communication Conference, San Francisco., CA.
[4] Hamid Ali Abed Al-Asadi, "Temperature dependence of the noise characteristics of Multiisection semiconductor lasers," Science Journal, vol. 7, No. 3, 2001.

[5] Hamid Ali Abed Al-Asadi, "Theoretical investigation of spectral linewidth properties of double fused $1.3 \mathrm{um}$ MQW-VCA in reflection and transition modes," Tikrit Journal for Pure Science, vol. 8, No. 2, 2002.

[6] Hamid Ali Abed Al-Asadi, "Linewidth characteristics of vertical cavity surface emitting lasers due to external optical feedback," Science Journal, vol. 8, 2001.

[7] Manjeshwar, A., and Agrawal. 2002, "APTEEN: A Hybrid Protocol for Efficient Routing and Comprehensive Information Retrieval in Wireless Sensor Networks", Proceedings of Issues in Wireless Networks and Mobile computing, FL., USA.

[8] Akkaya, K., and Younis, M., "A Survey of Routing Protocols in Wireless Sensor Networks", Elsevier AdHoc Network Journal, vol.3, no. 1, pp.325-349.

[9] Balakumar N., "Highly Distributed and Energy Efficient Clustering Algorithm for Wireless Sensor Networks", International Journal of Research -Granthaalayah, Volume 4, Number 9, September 2016.

[10] Jaswant, SR, Neelesh, G \& Neetu, S 2014, 'Energy efficient data communication approach in wireless sensor networks', International Journal of Advanced Smart Sensor Network Systems, vol. 4, no. 3, pp. 01-12.

[11] Jeong, H, Nam, CS, Jeong, YS \& Shin, DR 2008, 'A mobile agent based LEACH in wireless sensor network', Proceedings of the International Conference on Advanced Communication Technology, pp. 75-78. 